Pacific

Journal of

Mathematics

\title{
ON CRDAHA AND FINITE GENERAL LINEAR AND UNITARY GROUPS
}

\author{
BHAMA SRINIVASAN
}

Volume 279 No. 1-2

December 2015 


\title{
ON CRDAHA AND FINITE GENERAL LINEAR AND UNITARY GROUPS
}

\author{
BHAMA SRINIVASAN
}

Dedicated to the memory of Robert Steinberg

\begin{abstract}
We show a connection between Lusztig induction operators in finite general linear and unitary groups and parabolic induction in cyclotomic rational double affine Hecke algebras. Two applications are given: an explanation of a bijection result of Broué, Malle and Michel, and some results on modular decomposition numbers of finite general linear groups.
\end{abstract}

\section{Introduction}

Let $\Gamma_{n}$ be the complex reflection group $G(e, 1, n)$, the wreath product of $\mathcal{S}_{n}$ and $\mathbb{Z} / e \mathbb{Z}$, where $e>1$ is fixed for all $n$. Let $H\left(\Gamma_{n}\right)$ be the cyclotomic rational double affine Hecke algebra, or CRDAHA, associated with the complex reflection group $\Gamma_{n}$. The representation theory of the algebras $H\left(\Gamma_{n}\right)$ is related to the representation theory of the groups $\Gamma_{n}$, and thus to the modular representation theory of finite general linear groups $\operatorname{GL}(n, q)$ and unitary groups $U(n, q)$. In this paper we study this connection in the context of a recent paper of Shan and Vasserot [2012]. In particular we show a connection between Lusztig induction operators in general linear and unitary groups and certain operators in a Heisenberg algebra acting on a Fock space. We give two applications of this result, where $\ell$ is a prime not dividing $q$ and $e$ is the order of $q \bmod \ell$. The first is a connection via Fock spaces between an induction functor in CRDAHA described in [Shan and Vasserot 2012] and Lusztig induction, which gives an explanation for a bijection given by Broué, Malle and Michel [1993] and Enguehard [1992] between characters in an $\ell$-block of a finite general linear, unitary or classical group and characters of a corresponding complex reflection group. The second is an application to the $\ell$-modular theory of $\operatorname{GL}(n, q)$, describing some Brauer characters by Lusztig induction, for large $\ell$.

The paper is organized as follows. In Section 3 we state the results on CRDAHA from [Shan and Vasserot 2012] that we need. We introduce the category $\mathcal{O}(\Gamma)=$ $\bigoplus_{n \geq 0} \mathcal{O}\left(\Gamma_{n}\right)$ where $\mathcal{O}\left(\Gamma_{n}\right)$ is the category $\mathcal{O}$ of $H\left(\Gamma_{n}\right)$.

MSC2010: 20C33.

Keywords: CRDAHA, $\operatorname{GL}(n, q)$. 
In Section 4 we describe the $\ell$-block theory of $\operatorname{GL}(n, q)$ and $U(n, q)$. The unipotent characters in a unipotent block are precisely the constituents of a Lusztig induced character from an $e$-split Levi subgroup. Complex reflection groups arise when considering the defect groups of the blocks.

In Section 5 we introduce the Fock space and the Heisenberg algebra, and describe the connection between parabolic induction in CRDAHA and a Heisenberg algebra action on a Fock space given in [Shan and Vasserot 2012]. We have a Fock space $\mathcal{F}_{m, \ell}^{(s)}$ where $m, \ell>1$ are positive integers and $(s)$ is an $\ell$-tuple of integers. In [Shan and Vasserot 2012] a functor $a_{\mu}^{*}$, where $\mu$ is a partition, is introduced on the Grothendieck group $[\mathcal{O}(\Gamma)]$ and is identified with an operator $S_{\mu}$ of a Heisenberg algebra on the above Fock space.

The case $\ell=1$ is considered in Section 6. We consider a Fock space with a basis indexed by unipotent representations of general linear or unitary groups. We define the action of a Heisenberg algebra on this by a Lusztig induction operator $\mathcal{L}_{\mu}$ and prove that it can be identified with an operator $S_{\mu}$ defined by Leclerc and Thibon [1996]. This is one of the main results of the paper. It involves using a map introduced by Farahat [1954] on the characters of symmetric groups, which appears to be not widely known.

In Sections 7 and 8 we give applications of this result, using the results of Section 5. The first application is that parabolic induction $a_{\mu}^{*}$ in CRDAHA and Lusztig induction $\mathcal{L}_{\mu}$ on general linear or unitary groups can be regarded as operators arising from equivalent representations of the Heisenberg algebra. This gives an explanation for an observation of Broué, Malle and Michel on a bijection between Lusztig induced characters in a block of $\operatorname{GL}(n, q)$ and $U(n, q)$ and characters of a complex reflection group arising from the defect group of the block.

The second application deals with $\ell$-decomposition numbers of the unipotent characters of $\operatorname{GL}(n, q)$ for large $\ell$. Via the $q$-Schur algebra we can regard these numbers as arising from the coefficients of a canonical basis $G^{-}(\lambda)$ of a Fock space, where $\lambda$ runs through all partitions, in terms of the standard basis. The $G^{-}(\lambda)$ then express the Brauer characters of $\operatorname{GL}(n, q)$ in terms of unipotent characters. The $G^{-}(\lambda)$ are also described in terms of the $S_{\mu}$, and so we finally get that if $\lambda=\mu+e \alpha$ where $\mu^{\prime}$ is $e$-regular, the Brauer character parametrized by $\lambda$ is in fact a Lusztig induced generalized character.

\section{Notation}

We let $\mathcal{P}, \mathcal{P}_{n}, \mathcal{P}^{\ell}, \mathcal{P}_{n}^{\ell}$ denote the set of all partitions, the set of all partitions of $n \geq 0$, the set of all $\ell$-tuples of partitions, and the set of all $\ell$-tuples of partitions of integers $n_{1}, n_{2}, \ldots n_{\ell}$ such that $\sum n_{i}=n$, respectively.

If $\mathcal{C}$ is an abelian category, we write $[\mathcal{C}]$ for the complexified Grothendieck group of $\mathcal{C}$. 
We write $\lambda \vdash n$ if $\lambda$ is a partition of $n \geq 0$. The parts of $\lambda$ are denoted by $\left\{\lambda_{1}, \lambda_{2}, \ldots\right\}$. If $\lambda=\left\{\lambda_{i}\right\}, \mu=\left\{\mu_{i}\right\}$ are partitions, then $\lambda+\mu=\left\{\lambda_{i}+\mu_{i}\right\}$ and $e \lambda=\left\{e \lambda_{i}\right\}$ where $e$ is a positive integer.

\section{CRDAHA, complex reflection groups}

The main reference for this section is [Shan and Vasserot 2012]. We use the notation of Section 3.3 (page 967) of this paper.

Let $\Gamma_{n}=\mu_{\ell} 2 \mathcal{S}_{n}$, where $\mu_{\ell}$ is the group of $\ell$-th roots of unity in $\mathbb{C}$ and $\mathcal{S}_{n}$ is the symmetric group of degree $n$, so that $\Gamma_{n}$ is a complex reflection group. The category of finite-dimensional complex representations of $\Gamma_{n}$ is denoted by $\operatorname{Rep}\left(\mathbb{C} \Gamma_{n}\right)$. The irreducible modules in $\operatorname{Rep}\left(\mathbb{C} \Gamma_{n}\right)$ are known by a classical construction and denoted by $\bar{L}_{\lambda}$ where $\lambda \in \mathcal{P}_{n}^{\ell}$ (see for instance [Shan and Vasserot 2012, Equation (3.4), p. 968]). Let $R(\Gamma)=\bigoplus_{n \geq 0}\left[\operatorname{Rep}\left(\mathbb{C} \Gamma_{n}\right)\right]$.

Let $\mathfrak{H}$ be the reflection representation of $\Gamma_{n}$ and $\mathfrak{H}^{*}$ its dual. The cyclotomic rational double affine Hecke algebra or CRDAHA associated with $\Gamma_{n}$ is denoted by $H\left(\Gamma_{n}\right)$, and is the quotient of the smash product of $\mathbb{C} \Gamma_{n}$ and the tensor algebra of $\mathfrak{H} \oplus \mathfrak{H}^{*}$ by certain relations. The definition involves certain parameters (see [Shan and Vasserot 2012, p. 967]) which play a role in the results we quote from [Shan and Vasserot 2012], although we will not state them explicitly.

The category $\mathcal{O}$ of $H\left(\Gamma_{n}\right)$ is denoted by $\mathcal{O}\left(\Gamma_{n}\right)$. This is the category of $H\left(\Gamma_{n}\right)$ modules whose objects are finitely generated as $\mathbb{C}[\mathfrak{H}]$-modules and are $\mathfrak{H}$-locally nilpotent. Here $\mathbb{C}[\mathfrak{H}]$ is the subalgebra of $H\left(\Gamma_{n}\right)$ generated by $\mathfrak{H}^{*}$. Then $\mathcal{O}\left(\Gamma_{n}\right)$ is a highest weight category (see for instance [Rouquier et al. 2013]) and its standard modules are denoted by $\Delta_{\lambda}$ where $\lambda \in \mathcal{P}_{n}^{\ell}$. Let $\mathcal{O}(\Gamma)=\bigoplus_{n \geq 0} \mathcal{O}\left(\Gamma_{n}\right)$. This is one of the main objects of our study.

We then have a $\mathbb{C}$-linear isomorphism spe $:\left[\operatorname{Rep}\left(\mathbb{C} \Gamma_{n}\right)\right] \rightarrow\left[\mathcal{O}\left(\Gamma_{n}\right)\right]$ given by $\left[\bar{L}_{\lambda}\right] \rightarrow\left[\Delta_{\lambda}\right]$. We will from now on consider $\left[\mathcal{O}\left(\Gamma_{n}\right)\right]$ instead of $\left[\operatorname{Rep}\left(\mathbb{C} \Gamma_{n}\right)\right]$.

Let $r, m, n \geq 0$. For $n, r$ we have a parabolic subgroup $\Gamma_{n, r} \cong \Gamma_{n} \times \mathcal{S}_{r}$ of $\Gamma_{n+r}$, and there is a canonical equivalence of categories $\mathcal{O}\left(\Gamma_{n, r}\right)=\mathcal{O}\left(\Gamma_{n}\right) \otimes \mathcal{O}\left(\mathcal{S}_{r}\right)$ (for the tensor product of categories, see for instance [Deligne 1990, Section 5.1, Proposition 5.13]). By the work of Bezrukavnikov and Etingof [2009] there are induction and restriction functors

$$
{ }^{\mathcal{O}} \operatorname{Ind}_{n, r}: \mathcal{O}\left(\Gamma_{n}\right) \otimes \mathcal{O}\left(\mathcal{S}_{r}\right) \rightarrow \mathcal{O}\left(\Gamma_{n+r}\right)
$$

and

$$
{ }^{\mathcal{O}} \operatorname{Res}_{n, r}: \mathcal{O}\left(\Gamma_{n+r}\right) \rightarrow \mathcal{O}\left(\Gamma_{n}\right) \otimes \mathcal{O}\left(\mathcal{S}_{r}\right)
$$

For $\mu \vdash r$, Shan and Vasserot [2012, Section 5.1] defined functors $A_{\mu, !}, A_{\mu}^{*}, A_{\mu, *}$ on the bounded derived category $\mathcal{D}^{b}(\mathcal{O}(\Gamma))$. 
Here we will be concerned with $A_{\mu}^{*}$, defined as follows.

$$
\begin{aligned}
A_{\mu}^{*}: \mathcal{D}^{b}\left(\mathcal{O}\left(\Gamma_{n}\right)\right) & \rightarrow \mathcal{D}^{b}\left(\mathcal{O}\left(\Gamma_{n+m r}\right)\right), \\
M & \rightarrow{ }^{\mathcal{O}} \operatorname{Ind}_{n, m r}\left(M \otimes L_{m \mu}\right)
\end{aligned}
$$

Then $a_{\mu}^{*}$ is defined as the endomorphism of $[\mathcal{O}(\Gamma)]$ induced by $A_{\mu}^{*}$.

\section{Finite general linear and unitary groups}

In this section we describe a connection between the block theory of $\operatorname{GL}(n, q)$ or $U(n, q)$, and complex reflection groups. This was first observed by Broué, Malle and Michel [1993] and Enguehard [1992] for arbitrary finite reductive groups.

Let $G_{n}=\operatorname{GL}(n, q)$ or $U(n, q)$. The unipotent characters of $G_{n}$ are indexed by partitions of $n$. Using the description in [Broué et al. 1993, p. 45] we denote the character corresponding to $\lambda \vdash n$ of $\operatorname{GL}(n, q)$ or the character, up to sign, corresponding to $\lambda \vdash n$ of $U(n, q)$ as in [Fong and Srinivasan 1982] by $\chi_{\lambda}$.

Let $\ell$ be a prime not dividing $q$ and $e$ the order of $q \bmod \ell$. The $\ell$-modular representations of $G_{n}$ have been studied by various authors (see for instance [Cabanes and Enguehard 2004]) since they were introduced in [Fong and Srinivasan 1982]. The partition of the unipotent characters of $G_{n}$ into $\ell$-blocks is described in the following theorem from [Fong and Srinivasan 1982]. This classification depends only on $e$, so we can refer to an $\ell$-block as an $e$-block, e.g., in Section 7.

Theorem 4.1. The unipotent characters $\chi_{\lambda}$ and $\chi_{\mu}$ of $G_{n}$ are in the same e-block if and only if the partitions $\lambda$ and $\mu$ of $n$ have the same e-core.

There are subgroups of $G_{n}$ called $e$-split Levi subgroups ([Cabanes and Enguehard 2004, p. 190]). In the case of $G_{n}=\operatorname{GL}(n, q)$ an $e$-split Levi subgroup $L$ is of the form a product of smaller general linear groups over $F_{q^{e}}$ and $G_{k}$ with $k \leq n$. In the case of $G_{n}=U(n, q), L$ is of the form a product of smaller general linear groups or of smaller unitary groups over $F_{q^{e}}$ and $G_{k}$ with $k \leq n$. Then a pair $\left(L, \chi_{\lambda}\right)$ is an $e$-cuspidal pair if $L$ is $e$-split of the form a product of copies of tori, all of order $q^{e}-1$ in the case of $\operatorname{GL}(n, q)$, or all of orders $q^{e}-1, q^{2 e}-1$ or $q^{e / 2}+1$ in the case of $U(n, q)$ and $G_{k}$, where $G_{k}$ has an $e$-cuspidal unipotent character $\chi_{\lambda}$ ([Broué et al. 1993, p. 18, p. 27; Enguehard 1992, p. 42]). Here a character of $L$ is $e$-cuspidal if it is not a constituent of a character obtained by Lusztig induction $R_{M}^{L}$ from a proper $e$-split Levi subgroup $M$ of $L$.

The unipotent blocks, i.e., blocks containing unipotent characters, are classified by $e$-cuspidal pairs up to $G_{n}$-conjugacy. Let $B$ be a unipotent block corresponding to $\left(L, \chi_{\lambda}\right)$. Then if $\mu \vdash n, \chi_{\mu} \in B$ if and only if $\left\langle R_{L}^{G_{n}}\left(\chi_{\lambda}\right), \chi_{\mu}\right\rangle \neq 0$. As above, $R_{L}^{G_{n}}$ is Lusztig induction.

The defect group of a unipotent block is contained in $N_{G_{n}}(T)$ for a maximal torus $T$ of $G_{n}$ such that $N_{G_{n}}(T) / T$ is isomorphic to a complex reflection group 
$W_{G_{n}}(L, \lambda)=\mathbb{Z}_{e} \imath S_{k}$ for some $k \geq 1$. Here $\mathbb{Z}_{e}=\mathbb{Z} / e \mathbb{Z}$. Thus the irreducible characters of $W_{G_{n}}(L, \lambda)$ are parametrized by $\mathcal{P}_{k}^{e}$.

Let $B$ be a unipotent block of $G_{n}$ and $W_{G_{n}}(L, \lambda)$ as above. We then have the following theorem due to Broué, Malle and Michel [1993, Section 3.2] and to Enguehard [1992, Theorem B].

Theorem 4.2 (Global to local bijection for $G_{n}$ ). Let $M$ be an e-split Levi subgroup containing $L$ and let $W_{M}(L, \lambda)$ be defined as above for $M$. Let $\mu$ be a partition, and let $I_{L}^{M}$ be the isometry mapping the character of $W_{M}(L, \lambda)$ parametrized by the e-quotient of $\mu$ to the unipotent character $\chi_{\mu}$ of $M$ (up to sign) which is a constituent of $R_{M}^{G_{n}}(\lambda)$. Then we have $R_{M}^{G_{n}} I_{L}^{M}=I_{L}^{G_{n}} \operatorname{Ind}_{W_{M}(L, \lambda)}^{W_{G_{n}}(L, \lambda)}$.

The theorem is proved case by case for "generic groups", and thus for finite reductive groups. We have stated it only for $G_{n}$.

We state a refined version of the theorem involving CRDAHA and prove it in Section 7.

\section{Heisenberg algebra, Fock space}

Throughout this section we use the notation of [Shan and Vasserot 2012, Sections $4.2,4.5,4.6]$.

The affine Kac-Moody algebra $\widehat{\mathfrak{s} \ell_{\ell}}$ is generated by elements $e_{p}, f_{p}$ for $p=$ $0, \ldots, \ell-1$, satisfying Serre relations ([Shan and Vasserot 2012, Section 3.4]). We have $\widehat{\mathfrak{s} \ell_{\ell}}=\mathfrak{s} \ell_{\ell} \otimes \mathbb{C}\left[t, t^{-1}\right] \oplus \mathbb{C} \mathbf{1}$, where $\mathbf{1}$ is central.

The Heisenberg algebra is the Lie algebra $\mathfrak{H}$ generated by $1, b_{r}, b_{r}^{\prime}$ for $r \geq 0$, with relations $\left[b_{r}^{\prime}, b_{s}^{\prime}\right]=\left[b_{r}, b_{s}\right]=0,\left[b_{r}^{\prime}, b_{s}\right]=r 1 \delta_{r, s}$ for $r, s \geq 0$ ([Shan and Vasserot 2012, Section 4.2]). In $U(\mathfrak{H})$ we then have elements $b_{r_{1}}, b_{r_{2}}, \ldots$ with $\sum_{i} r_{i}=r$. If $\lambda \in \mathcal{P}$ we then have the element $b_{\lambda}=b_{\lambda_{1}} b_{\lambda_{2}} \ldots$, and then for any symmetric function $f$ the element $b_{f}$ equals $\sum_{\lambda \in \mathcal{P}} z_{\lambda}^{-1}\left\langle P_{\lambda}, f\right\rangle b_{\lambda}$. Here $P_{\lambda}$ is a power sum symmetric function and $z_{\lambda}=\prod_{i} i^{m_{i}} m_{i}$ ! where $m_{i}$ is the number of parts of $\lambda$ equal to $i$. The scalar product $\langle\cdot, \cdot\rangle$ is the one used in symmetric functions, where the Schur functions form an orthonormal basis (see [Macdonald 1995]).

We now define Fock spaces $\mathcal{F}_{m}, \mathcal{F}_{m, \ell}^{(d)}$ and $\mathcal{F}_{m, \ell}^{(s)}$, where $m>1$. Choose a basis $\left(\epsilon_{1}, \ldots, \epsilon_{m}\right)$ of $\mathbb{C}^{m}$. If $d \in \mathbb{Z}$, let $\mathcal{F}_{m}^{(d)}$ be the space of elements of the form $u_{i_{1}} \wedge u_{i_{2}} \ldots$ for $i_{1}>i_{2} \ldots$, where $u_{i-j m}=\epsilon_{i} \otimes t^{j}$ with $i_{k}=d-k+1$ for $k \gg 0$. If we set $|\lambda, d\rangle=u_{i_{1}} \wedge u_{i_{2}} \ldots$ for $i_{k}=\lambda_{k}+d-k+1$, the elements $|\lambda, d\rangle$ with $\lambda \in \mathcal{P}$ form a basis of $\mathcal{F}_{m}^{(d)}$. The Fock space $\mathcal{F}_{m}$ is defined as the space of semi-infinite wedges of the $\mathbb{C}$-vector space $\mathbb{C}^{m} \otimes \mathbb{C}\left[t, t^{-1}\right]$, and we have $\mathcal{F}_{m}=\bigoplus_{d \in \mathbb{Z}} \mathcal{F}_{m}{ }^{(d)}$. Then $\widehat{\mathfrak{s} \ell_{m}}$ acts on $\mathcal{F}_{m}{ }^{(d)}$. This setup has been studied by Leclerc and Thibon [1996; 2000].

Similarly choose a basis $\left(\epsilon_{1}, \ldots \epsilon_{m}\right)$ of $\mathbb{C}^{m}$ and a basis $\left(\epsilon_{1}^{\prime}, \ldots, \epsilon_{\ell}^{\prime}\right)$ of $\mathbb{C}^{\ell}$.

The Fock space $\mathcal{F}_{m, \ell}$ of rank $m$ and level $\ell$ is defined as the space of semi-infinite wedges, i.e., elements of the form $u_{i_{1}} \wedge u_{i_{1}} \ldots$ with $i_{1}>i_{2}>\ldots$, where the $u_{j}$ are 
vectors in a $\mathbb{C}$-vector space $\mathbb{C}^{m} \otimes \mathbb{C}^{\ell} \otimes \mathbb{C}\left[z, z^{-1}\right]$ given by $u_{i+(j-1) m-k m \ell}=\epsilon_{i} \otimes \epsilon_{j}^{\prime} \otimes z^{k}$, with $i=1,2, \ldots m, j=1,2, \ldots \ell, k \in \mathbb{Z}$. Then $\widehat{\mathfrak{s} \ell_{\ell}}, \widehat{\mathfrak{s} \ell_{m}}$ and $\mathfrak{H}$ act on the space ([Shan and Vasserot 2012, Section 4.6]), and these actions are pairwise commuting.

Let $d \in \mathbb{Z}$. There is a space $\Lambda^{d+\infty / 2}$ defined by Uglov [2000, Section 4.1]. This space has a basis which Uglov indexes by $\mathcal{P}$ or by pairs $(\lambda, s)$ where $\lambda \in \mathcal{P}^{m}$ and $s=\left(s_{p}\right)$ is an $m$-tuple of integers with $\sum_{p} s_{p}=d$. There is a bijection between the two index sets given by $\lambda \rightarrow\left(\lambda^{*}, s\right)$ where $\lambda^{*}$ is the $m$-quotient of $\lambda$ and $s$ is a particular labeling of the $m$-core of $\lambda$ ([Uglov 2000, Sections 4.1, 4.2]).

We have a decomposition $\mathcal{F}_{m, \ell}=\bigoplus_{d \in \mathbb{Z}} \mathcal{F}_{m, \ell}^{(d)}$ defined using semi-infinite wedges, as in the case of $\mathcal{F}_{m}$. Then $\mathcal{F}_{m, \ell}^{(d)}$ can be identified with the space defined by Uglov.

There is a subspace $\mathcal{F}_{m, \ell}^{(s)}$ of $\mathcal{F}_{m, \ell}^{(d)}$, the Fock space associated with $(s)$, which is a weight space for the $\mathfrak{s} \ell \ell_{\ell}$ action ([Shan and Vasserot 2012, p. 982]). We have $\mathcal{F}_{m, \ell}^{(d)}=\bigoplus \mathcal{F}_{m, \ell}^{(s)}$, the sum of weight spaces. Here we can define a basis $\{|\lambda, s\rangle\}$ with $\lambda \in \mathcal{P}^{\ell}$ of $\mathcal{F}_{m, \ell}$. The spaces $\mathcal{F}_{m, \ell}^{(s)}$ were also studied by Uglov.

The endomorphism of $\mathbb{C}^{m} \otimes \mathbb{C}\left[t, t^{-1}\right]$ induced by multiplication by $t^{r}$ gives rise to a linear operator $b_{r}$ and its adjoint $b_{r}^{\prime}$ on $\mathcal{F}_{m}{ }^{(d)}$, and thus to an action of $\mathfrak{H}$ on $\mathcal{F}_{m}{ }^{(d)}$. We also have an action of $\mathfrak{H}$ by operators $b_{r}, b_{r}^{\prime}$ on $\mathcal{F}_{m, \ell}{ }^{(s)}$, and this is the main result that we need ([Shan and Vasserot 2012, p. 982]).

We now choose a fixed $\ell$-tuple $s$. With suitable parameters of $H\left(\Gamma_{n}\right)$ for each $n$, the $\mathbb{C}$-vector space $[\mathcal{O}(\Gamma)]$ is then canonically isomorphic to $\mathcal{F}_{m, \ell}^{(s)}$. We then have the following $\mathbb{C}$-linear isomorphisms ([Shan and Vasserot 2012, Equation (5.20), p. 990]):

$$
\begin{aligned}
{[\mathcal{O}(\Gamma)] } & \rightarrow R(\Gamma) \rightarrow \mathcal{F}_{m, \ell}^{(s)}, \\
\Delta_{\lambda} & \rightarrow \bar{L}_{\lambda} \rightarrow|\lambda, s\rangle .
\end{aligned}
$$

Consider the Fock space $\mathcal{F}_{m, \ell}^{(s)}$ with basis indexed by $\{|\lambda, s\rangle\}$ where $\lambda \in \mathcal{P}^{\ell}$. The element $b_{s_{\mu}} \in \mathfrak{H}$, i.e., $b_{f}$ where $f=s_{\mu}$, a Schur function, acts by an operator $S_{\mu}$ on the space. The functor $a_{\mu}^{*}$ on $[\mathcal{O}(\Gamma)]$ (see Section 3) is now identified with $S_{\mu}$ by [Shan and Vasserot 2012, Proposition 5.13, p. 990].

Remark. The bijection between $m$-core partitions and the $m$-tuples $(s)$ as above has been studied by combinatorialists (see for instance [Garvan et al. 1990]).

\section{Fock space revisited}

References for the combinatorial definitions in this section are [Leclerc and Thibon 1996; 2000]. Given a partition $\mu$ we introduce three operators on a Fock space: an operator $S_{\mu}$ defined by Leclerc and Thibon [1996], an operator $\mathcal{F}_{\mu}^{*}$ defined by Farahat [1954] on representations of the symmetric groups $\mathcal{S}_{n}$, and the operators $\mathcal{L}_{\mu}$ of Lusztig induction on $G_{n}$. The algebra of symmetric functions in $\left\{x_{1}, x_{2}, \ldots\right\}$ is denoted by $\Lambda$. 
Integers $\ell, m$ were introduced in Section 5. For the rest of the paper we set $\ell=m=e$, where $e$ is a positive integer which was used in the context of blocks of $G_{n}$. Thus $\Gamma_{n}=\mu_{e}$ 2 $\mathcal{S}_{n}$.

First consider the space $\mathcal{F}_{e}^{(d)}$ where $d \in \mathbb{Z}$, with basis elements $\{|\lambda, d\rangle\}$ where $\lambda \in \mathcal{P}$. Leclerc and Thibon [1996] introduced elements in $U(\mathfrak{H})$ which we write in our previous notation as $b_{h_{\rho}}$ and $b_{s_{\mu}}$, acting as operators $V_{\rho}$ and $S_{\mu}$ on $\mathcal{F}_{e}^{(d)}$ where $\rho, \mu \in \mathcal{P}$ and $h_{\rho}$ is a homogeneous symmetric function. These operators have a combinatorial description as follows. Here we will write $|\lambda\rangle$ for $|\lambda, d\rangle$.

First they define commuting operators $V_{k}$ for $k \geq 1$ on $\mathcal{F}_{e}^{(d)}$ defined by

$$
V_{k}(|\lambda\rangle)=\sum_{\mu}(-1)^{-s(\mu / \lambda)}|\mu\rangle
$$

where the sum is over all $\mu$ such that $\mu / \lambda$ is a horizontal $n$-ribbon strip of weight $k$, and $s(\mu / \lambda)$ is the "spin" of the strip.

Remark. The minus sign in the exponent in the formula is not necessary, but appears because it is a special case of a quantized formula.

Here a ribbon is the same as a rim-hook, i.e., a skew partition which does not contain a $2 \times 2$ square. The spin is the leg length of the ribbon, i.e., the number of rows -1 .

Definition. (see [Lam 2005]) A horizontal $n$-ribbon strip of weight $k$ is a tiling of a skew partition by $k n$-ribbons such that the top rightmost square of every ribbon touches the northern edge of the shape. The spin of the strip is the sum of the spins of all the ribbons.

It can be shown that a tiling of a skew partition as above is unique. More generally we can then define $V_{\rho}$ where $\rho$ is a composition. If $\rho=\left\{\rho_{1}, \rho_{2}, \ldots\right\}$ then $V_{\rho}=V_{\rho_{1}} . V_{\rho_{2}} \ldots$. Finally we define operators $S_{\mu}$ acting on $\mathcal{F}_{e}^{(d)}$ which we connect to Lusztig induction. They coincide with the operators mentioned at the end of the last section.

Definition. We have $S_{\mu}=\sum_{\rho} \kappa_{\mu \rho} V_{\rho}$ where the $\kappa_{\mu \rho}$ are inverse Kostka numbers ([Leclerc and Thibon 1996, p. 204; Lam 2005, p. 8]).

Remark. Let $p_{e}(f)$ denote the plethysm by the power function in $\Lambda$, i.e.,

$$
p_{e}\left(f\left(x_{1}, x_{2}, \ldots\right)\right)=f\left(x_{1}^{e}, x_{2}^{e}, \ldots\right) .
$$

(This is related to a Frobenius morphism; see [Leclerc and Thibon 2000, p. 171].) In fact in [Leclerc and Thibon 1996] $\mathfrak{H}$ is regarded as a $\mathbb{C}(v)$-space where $v$ is an indeterminate. Then $V_{\rho}$ and $S_{\mu}$ are $v$-analogs of multiplication by $p_{e}\left(h_{\rho}\right)$ and $p_{e}\left(s_{\mu}\right)$ in $\Lambda$. 
Next, let $\mathcal{A}_{n}$ be the category of unipotent representations of $G_{n}$. Let $\mathcal{A}=$ $\bigoplus_{n \geq 0}\left[\mathcal{A}_{n}\right]$. We recall from Section 4 that the unipotent characters of $G_{n}$ are denoted by $\left\{\chi_{\lambda}\right\}$ where $\lambda \vdash n$. We now regard $\mathcal{A}$ as having a basis $\left[\chi_{\lambda}\right]$ where $\lambda$ runs through all partitions. Then $\mathcal{A}$ is isomorphic to $\mathcal{F}_{e}^{(d)}$ as a $\mathbb{C}$-vector space, since $\mathcal{A}$ also has a basis indexed by partitions.

We now define Lusztig operators $\mathcal{L}_{\mu}$ on $\mathcal{A}$ and then relate them to the $S_{\mu}$.

Definition. Let $\mu \vdash k$. The Lusztig map $\mathcal{L}_{\mu}: \mathcal{A} \rightarrow \mathcal{A}$ is as follows. Define $\mathcal{L}_{\mu}:\left[\mathcal{A}_{n}\right] \rightarrow\left[\mathcal{A}_{n+k e}\right]$ by $\left[\chi_{\lambda}\right] \rightarrow\left[R_{L}^{G_{n+k e}}\left(\chi_{\lambda} \times \chi_{\mu}\right)\right]$, where $L=G_{n} \times \operatorname{GL}\left(k, q^{e}\right)$ or $L=G_{n} \times U\left(k, q^{e}\right)$, an $e$-split Levi subgroup of $G_{n+k e}$.

Finally, consider the characters of $\mathcal{S}_{n}$. We denote the character corresponding to $\lambda \in \mathcal{P}_{n}$ as $\phi_{\lambda}$. We also use $\lambda \in \mathcal{P}_{n}$ to denote representatives of conjugacy classes of $\mathcal{S}_{n}$. Let $\mathcal{C}_{n}$ be the category of representations of $\mathcal{S}_{n}$ and $\mathcal{C}=\bigoplus_{n \geq 0}\left[\mathcal{C}_{n}\right]$.

Given partitions $v \vdash(n+k e), \lambda \vdash n$ such that $v / \lambda$ is defined, Farahat [1954] has defined a character $\hat{\phi}_{v / \lambda}$ of $S_{k}$, as follows. Let the $e$-tuples $\left(v^{(i)}\right),\left(\lambda^{(i)}\right)$ be the $e$-quotients of $v$ and $\lambda$. Then $\epsilon \prod_{i} \phi_{\left(v^{(i)} / \lambda^{(i)}\right)}$, where $\epsilon= \pm 1$ is a character of a Young subgroup of $S_{k}$, which induces up to the character $\hat{\phi}_{v / \lambda}$ of $S_{k}$.

We will instead use an approach of Enguehard ([1992, p. 37]) which is more conceptual and convenient for our purpose.

Definition. The Farahat map $\mathcal{F}:\left[\mathcal{C}_{e k}\right] \rightarrow\left[\mathcal{C}_{k}\right]$ is defined by $(\mathcal{F} \chi)(\mu)=\chi(e \mu)$, where $\mu \vdash k$.

Let $\mu \vdash k$. Taking adjoints and denoting $\mathcal{F}^{*}$ by $\mathcal{F}_{\mu}^{*}$ we then have, for $\lambda \vdash n$ :

Definition. Define $\mathcal{F}_{\mu}^{*}:\left[\mathcal{C}_{n}\right] \rightarrow\left[\mathcal{C}_{n+e k}\right]$ by $\phi_{\lambda} \rightarrow \operatorname{Ind}_{\mathcal{S}_{e k} \times \mathcal{S}_{n}}^{\mathcal{S}_{n+e k}}\left(\mathcal{F}^{*}\left(\phi_{\mu}\right) \times \phi_{\lambda}\right)$.

By the standard classification of maximal tori in $G_{n}$ we can denote a set of representatives of the $G_{n}$-conjugacy classes of the tori by $\left\{T_{w}\right\}$, where $w$ runs over a set of representatives for the conjugacy classes of $S_{n}$. We then have that the unipotent character $\chi_{\lambda}=\frac{1}{\left|S_{n}\right|} \sum_{w \in S_{n}} \lambda(w) R_{T_{w}}^{G_{n}}(1)$ (see for instance [Fong and Srinivasan 1982, Equation (1.13)]). Here, as before, $R_{T_{w}}^{G_{n}}(1)$ is Lusztig induction.

We assume in the proposition below that when $G_{n}=U(n, q)$ that $e \equiv 0 \bmod 4$. This is the case that is analogous to the case of $\operatorname{GL}(n, q)$. The other cases for $e$ require some straightforward modifications which we mention below. The proof of the proposition has been sketched by Enguehard ([1992, p. 37]) when $G_{n}=\operatorname{GL}(n, q)$.

Let $M$ be the $e$-split Levi subgroup of $G_{n}$ isomorphic to $\operatorname{GL}\left(k, q^{e}\right) \times G L_{\ell}$. We denote by ${ }^{*} R_{M}^{G_{n}}$ the adjoint of the Lusztig map $R_{M}^{G_{n}}$. It is an analogue of the map $\mathcal{F}^{*}$, and this is made precise below.

If $\lambda \vdash n$, we have a bijection $\phi_{\lambda} \leftrightarrow \chi_{\lambda}$ between $\left[\mathcal{C}_{n}\right]$ and $\left[\mathcal{A}_{n}\right]$. We then have an obvious bijection $\psi: \phi_{\lambda} \leftrightarrow \chi_{\lambda}$ between $\mathcal{C}$ and $\mathcal{A}$. 
Proposition. Let $G=\mathrm{GL}(e k, q)$ or $U(e k, q)$. In the case of $U(e k, q)$ we assume $e \equiv 0 \bmod 4$. Let $M \cong \mathrm{GL}\left(k, q^{e}\right)$, a subgroup of $G$. Let $\psi: \phi_{\lambda} \leftrightarrow \chi_{\lambda}$ between $\mathcal{C}$ and $\mathcal{A}$ be as above. Then:

(i) If $\lambda \vdash e k$, then $\psi\left(\mathcal{F}\left(\phi_{\lambda}\right)\right)={ }^{*} R_{M}^{G}\left(\chi_{\lambda}\right)$.

(ii) If $\mu \vdash k$, then $\psi\left(\mathcal{F}^{*}\left(\phi_{\mu}\right)\right)=R_{M}^{G}\left(\chi_{\mu}\right)$.

Proof. We have

$$
\psi\left(\mathcal{F}\left(\phi_{\lambda}\right)\right)=\frac{1}{\left|\mathcal{S}_{k}\right|} \sum_{w \in \mathcal{S}_{k}}\left(\mathcal{F} \phi_{\lambda}\right)(w) R_{T_{w}}^{M}(1)=\frac{1}{\left|\mathcal{S}_{k}\right|} \sum_{w \in \mathcal{S}_{k}} \phi_{\lambda}(e w) R_{T_{w}}^{M}(1) .
$$

Since the torus parametrized by $w$ in $M$ is parametrized by $e w$ in $G$, we can write this as $\frac{1}{\left|\mathcal{S}_{k}\right|} \sum_{w \in \mathcal{S}_{k}} \phi_{\lambda}(e w) R_{T_{e w}}^{M}(1)$.

On the other hand, we have (see [Fong and Srinivasan 1982, Lemma 2B]), using the parametrization of tori in $M,{ }^{*} R_{M}^{G}\left(\chi_{\lambda}\right)=\frac{1}{\left|\mathcal{S}_{k}\right|} \sum_{w \in \mathcal{S}_{k}} \phi_{\lambda}(w) R_{T_{w}}^{M}(1)$. This proves (i). Then (ii) follows by taking adjoints.

The proposition clearly generalizes to the subgroup $M \cong \mathrm{GL}\left(k, q^{e}\right) \times G_{\ell}$ of $G_{n}$ where $n=e k+\ell$. In the case of $U(n, q)$, if $e$ is odd we replace $e$ by $e^{\prime}$ where $e^{\prime}=2 e$ with $M \cong \mathrm{GL}\left(k, q^{e^{\prime}}\right)$, and if $e \equiv 2 \bmod 4$ by $e^{\prime}$ where $e^{\prime}=e / 2$ with $M \cong U\left(k, q^{e^{\prime}}\right)$, the proof being similar.

Using the isomorphisms between the spaces $\mathcal{A}, \mathcal{C}$ and $\mathcal{F}_{e}^{(d)}$, we now regard the operators $\mathcal{L}_{\mu}, \mathcal{F}_{\mu}^{*}$ and $\mathcal{S}_{\mu}$ as acting on $\mathcal{F}_{e}^{(d)}$.

We now prove one of the main results in this paper.

Theorem 6.1. The operators $\mathcal{L}_{\mu}$ and $S_{\mu}$ on $\mathcal{F}_{e}^{(d)}$ coincide.

Proof. We note that $\mathcal{F}_{\mu}^{*}=\mathcal{L}_{\mu}$. This follows from the previous proposition, generalized to $G_{n}$, and the fact that parabolic induction in symmetric groups is compatible with Lusztig induction in $G_{n}$, using the combinatorial description of both functors. We will now show that $\mathcal{F}_{\mu}^{*}=S_{\mu}$.

More generally we consider the character $\hat{\phi}_{\nu / \lambda}$ of $S_{k}$ defined by Farahat, where $v \vdash(n+k e)$ and $\mu \vdash n$, and describe it using $\mathcal{F}$. The restriction of $\phi_{\nu}$ to $S_{n} \times S_{k e}$ can be written as a sum of $\phi_{\lambda} \times \phi_{\nu / \lambda}$ where $\phi_{\nu / \lambda}$ is a (reducible) character of $S_{k e}$, and characters not involving $\phi_{\lambda}$. We then define $\hat{\phi}_{v / \lambda}=\mathcal{F}\left(\phi_{\nu / \lambda}\right)$, a character of $S_{k}$. We then note that $\hat{\phi}_{v / \lambda}(u)=\phi_{\nu / \lambda}(e u)$. Using the characteristic map we get a corresponding skew symmetric function $s_{v^{*} / \lambda^{*}}$. This is precisely the function which has been described in [Macdonald 1995, p. 91], since it is derived from the usual symmetric function $s_{v / \lambda}$ by taking $e$-th roots of variables. Using the plethysm function $p_{e}$ and its adjoint $\psi_{e}$ ([Lascoux et al. 1997, p. 1048]) we get $s_{v^{*} / \lambda^{*}}=\psi_{e}\left(s_{v / \lambda}\right)$. 
By the above facts we get

$$
\begin{aligned}
\left(\hat{\phi}_{\nu / \lambda}, \phi_{\mu}\right) & =\left(s_{v^{*} / \lambda^{*}}, s_{\mu}\right)=\left(\psi_{e}\left(s_{v / \lambda}\right), s_{\mu}\right) \\
& =\left(s_{v / \lambda}, p_{e}\left(s_{\mu}\right)\right)=\left(p_{e}\left(s_{\mu}\right) \cdot s_{\lambda}, s_{\nu}\right) \\
& =\left(S_{\mu}\left[\chi_{\lambda}\right],\left[\chi_{\nu}\right]\right) .
\end{aligned}
$$

The last equality can be seen as follows. There is a $\mathbb{C}$-linear isomorphism between the algebra $\Lambda$ and $\mathcal{F}_{e}^{(d)}$, since both have bases indexed by $\mathcal{P}$. Under this isomorphism multiplication by the symmetric function $p_{e}\left(s_{\mu}\right)$ on $\Lambda$ corresponds to the operator $S_{\mu}$ on a Fock space (see [Leclerc and Thibon 1996, p. 6]).

This proves that $\mathcal{L}_{\mu}=S_{\mu}$.

We recall that $\widehat{s l}_{e}$ acts on $\mathcal{F}_{e}^{(d)}$ and hence on $\mathcal{A}$.

Corollary. The highest weight vectors $V_{\rho} \varnothing$ of the irreducible components of the $\widehat{s l}_{e}$-module $\mathcal{A}$ ([Lascoux et al. 1997, p. 1054]) can be described by Lusztig induction.

Remark. In fact Leclerc and Thibon also have a parameter $q$ in their definition of $S_{\mu}$, since they deal with a deformed Fock space. Thus $S_{\mu}$ can be regarded as a quantized version of a Lusztig operator $\mathcal{L}_{\mu}$.

Remark. In the notation of [Leclerc and Thibon 2000, p. 173] we have

$$
\left(s_{v^{*} / \lambda^{*}, s_{\mu}}\right)=\left(s_{v_{0} / \lambda_{0}} s_{v_{1} / \lambda_{1}} \ldots s_{v_{e-1} / \lambda_{e-1}}, s_{\mu}\right)=c_{\nu / \lambda}^{\mu} .
$$

In this equation, the $c_{v / \lambda}^{\mu}$ are Littlewood-Richardson coefficients. We now have $\left(\chi_{\nu}, R_{M}^{G_{n}}\left(\chi_{\lambda} \times \chi_{\mu}\right)\right)=\epsilon c_{v / \lambda}^{\mu}$, where $\epsilon= \pm 1$. In particular $c_{v / \lambda}^{(k)}$ is the number of tableaux of shape $v$ such that $v / \lambda$ is a horizontal $e$-ribbon of weight $k$. Thus the Lusztig operator $\mathcal{L}_{k}$ can be described in terms of $e$-ribbons of weight $k$, similar to the case of $k=1$ which classically is described by $e$-hooks.

\section{CRDAHA and Lusztig induction}

The main reference for parabolic induction in this section is [Shan and Vasserot 2012].

In this section we show a connection between the parabolic induction functor $a_{\mu}^{*}$ on $[\mathcal{O}(\Gamma)]$ and the Lusztig induction functor $\mathcal{L}_{\mu}$ in $\mathcal{A}$ using Fock spaces. In particular this gives an explanation of the global to local bijection for $G_{n}$ given in Theorem 4.2. This can be regarded as a local, block-theoretic version of Theorem 6.1.

As mentioned in Section 4, the unipotent characters $\chi_{\lambda}$ in an $e$-block of $G_{n}$ are constituents of the Lusztig character $R_{L}^{G_{n}}(\lambda)$ where $(L, \lambda)$ is an $e$-cuspidal pair. Up to sign, they are in bijection with the characters of $W_{G_{n}}(L, \lambda)$, and they all have the same $e$-core. 
For our result we can assume $d=0$, which we do from now on. We set $\ell=m=e$ as in Section 6. We have spaces $\mathcal{F}_{e}^{(0)}$ and $\mathcal{F}_{e, e}^{(0)}=\bigoplus_{s} \mathcal{F}_{e, e}^{(s)}$ where $s=\left(s_{p}\right)$ is an $e$-tuple of integers with $\sum_{p} s_{p}=0$. We now fix such an $s$.

By [Shan and Vasserot 2012, Sections 6.17, 6.22, p. 1010] we have an $U(\mathfrak{H})$ isomorphism between $\mathcal{F}_{e}^{(0)}$ and $\mathcal{F}_{e, e}^{(0)}$. Let $\mathcal{F}_{e}^{(s)}$ be the inverse image of $\mathcal{F}_{e, e}^{(s)}$ under this isomorphism. We then have $\mathbb{C}$-isomorphisms from $\mathcal{F}_{e, e}^{(s)}$ to $[\mathcal{O}(\Gamma)]$, and from $\mathcal{F}_{e}^{(s)}$ to $\mathcal{A}^{(s)}$, where $\mathcal{A}^{(s)}$ is the subspace of $\mathcal{A}$ spanned by $\left[\chi_{\lambda}\right]$ where the $\chi_{\lambda}$ are in $e$-blocks parametrized by $e$-cores labeled by $(s)$ (see Section 5 ).

The spaces $\mathcal{F}_{e}^{(s)}, \mathcal{F}_{e, e}^{(s)},[\mathcal{O}(\Gamma)], \mathcal{A}^{(s)}$ have bases $\{|\lambda, s\rangle: \lambda \in \mathcal{P}\},\left\{|\lambda, s\rangle: \lambda \in \mathcal{P}^{e}\right\}$, $\left\{\Delta_{\lambda}: \lambda \in \mathcal{P}^{e}\right\}$ and $\left[\chi_{\lambda}\right]$ where $\lambda$ has $e$-core labeled by $s$, respectively.

We have maps $S_{\mu}: \mathcal{F}_{e, e}^{(s)} \rightarrow \mathcal{F}_{e, e}^{(s)}$ for $\mu \in \mathcal{P}^{e}, S_{\mu}: \mathcal{F}_{e}^{(s)} \rightarrow \mathcal{F}_{e}^{(s)}$ for $\mu \in \mathcal{P}$, $\mathcal{L}_{\mu}: \mathcal{A}^{(s)} \rightarrow \mathcal{A}^{(s)}$ and $a_{\mu}^{*}:[\mathcal{O}(\Gamma)] \rightarrow[\mathcal{O}(\Gamma)]$.

Here we note that Lusztig induction preserves $e$-cores, and thus $\mathcal{L}_{\mu}$ fixes $\mathcal{A}^{(s)}$.

The following theorem can be regarded as a refined version of the global to local bijection of [Broué et al. 1993]. The case $e=1$ is due to Enguehard ([1992, p. 37]), where the proof is a direct verification of the theorem from the definition of the Farahat map $\mathcal{F}$ in $\mathcal{S}_{n}$ (see Section 6) and Lusztig induction in $G_{n}$.

Theorem 7.1. Under the isomorphism $\mathcal{A}^{(s)} \cong[\mathcal{O}(\Gamma)]$ given by $\left[\chi_{\lambda}\right] \rightarrow\left[\Delta_{\lambda^{*}}\right]$ where $\lambda^{*}$ is the e-quotient of $\lambda$, Lusztig induction $\mathcal{L}_{\mu}$ on $\mathcal{A}^{(s)}$ with $\mu \in \mathcal{P}$ corresponds to parabolic induction $a_{\mu}^{*}$ on $[\mathcal{O}(\Gamma)]$ with $\mu \in \mathcal{P}^{e}$.

Proof. Consider the action of $b_{s_{\mu}} \in U(\mathfrak{H})$ on $\mathcal{F}_{e, e}^{(s)}$. The operator $S_{\mu}$ acting on $\mathcal{F}_{e, e}^{(s)}$ can be identified with $a_{\mu}^{*}$ acting on $[\mathcal{O}(\Gamma)]$, with the basis element $|\lambda, s\rangle$ corresponding to $\left[\Delta_{\lambda}\right]$ ([Shan and Vasserot 2012, Equation (5.20)]).

On the other hand, $b_{s_{\mu}} \in U(\mathfrak{H})$ acts as $S_{\mu}$ on the space $\mathcal{F}_{e}^{(s)}$ and thus, by Theorem 6.1 as $\mathcal{L}_{\mu}$ on $\mathcal{A}^{(s)}$ with the basis element $|\lambda, s\rangle$ corresponding to $\left[\chi_{\lambda}\right]$.

Now $\mathcal{F}_{e}^{(s)}$ is isomorphic to $\mathcal{A}^{(s)}$ and $\mathcal{F}_{e, e}^{(s)}$ is isomorphic to $[\mathcal{O}(\Gamma)]$. Thus we have shown that $a_{\mu}^{*}$ and $\mathcal{L}_{\mu}$ correspond under two equivalent representations of $U(\mathfrak{H})$.

Corollary. The BMM-bijection of Theorem 4.2 between the constituents of the Lusztig map $R_{L}^{G_{n}}(\lambda)$ where $(L, \lambda)$ is an e-cuspidal pair and the characters of $W_{G_{n}}(L, \lambda)$ is described via equivalent representations of $U(\mathfrak{H})$ on Fock spaces.

This follows from the theorem, using the map spe (see Section 3).

\section{Decomposition numbers}

References for this section are [Dipper and James 1989; Leclerc and Thibon 1996; 2000]. In this section we assume $G_{n}=\operatorname{GL}(n, q)$, since we will be using the connection with $q$-Schur algebras. We describe connections between weight spaces of $\widehat{\mathfrak{s} \ell_{e}}$ on Fock spaces, blocks of $q$-Schur algebras, and blocks of $G_{n}$. We show that some Brauer characters of $G_{n}$ can be described by Lusztig induction. 
The $\ell$-decomposition numbers of the groups $G_{n}$ have been studied by Dipper and James and by Geck, Gruber, Hiss and Malle. The latter have also studied the classical groups, using modular Harish-Chandra induction. One of the key ideas in these papers is to compare the decomposition matrices of the groups with those of $q$-Schur algebras.

We have the Dipper-James theory over a field of characteristic 0 or $\ell$. Dipper and James define ([1989, Section 2.9]) the $q$-Schur algebra $\mathcal{S}_{q}(n)$, endomorphism algebra of a sum of permutation representations of the Hecke algebra $\mathcal{H}_{n}$ of type $A_{n-1}$. The unipotent characters and the $\ell$-modular Brauer characters of $G_{n}$ are both indexed by partitions of $n$ (see [Fong and Srinivasan 1982]). Similarly the Weyl modules and the simple modules of $\mathcal{S}_{q}(n)$ are both indexed by partitions of $n$ (see [Dipper and James 1989]).

For $\mathcal{S}_{q}(n)$ over $k$ of characteristic $\ell, q \in k$, one can define the decomposition matrix of $\mathcal{S}_{q}(n)$, where $q$ is an $e$-th root of unity, where as before $e$ is the order of $q \bmod \ell$. By the above, this is a square matrix whose entries are the multiplicities of simple modules in Weyl modules. Dipper and James ([1989, Theorem 4.9]) showed that this matrix, up to reordering the rows and columns, is the same as the unipotent part of the $\ell$-decomposition matrix of $G_{n}$, the transition matrix between the ordinary (complex) characters and the $\ell$-modular Brauer characters. The rows and columns of the matrices are indexed by partitions of $n$.

We consider the Fock space $\mathcal{F}=\mathcal{F}_{e}{ }^{(d)}$ for a fixed $d$, which as in Section 6 is isomorphic to $\mathcal{A}$, and has the standard basis $\{|\lambda\rangle: \lambda \in \mathcal{P}\}$. It also has two canonical bases $G^{+}(\lambda)$ and $G^{-}(\lambda)$ for $\lambda \in \mathcal{P}$ ([Leclerc and Thibon 1996; 2000]). There is a recursive algorithm to determine these two bases.

We fix an $s$ as in Section 6. The algebra $\widehat{\mathfrak{s} \ell_{e}}$ acts on $\mathcal{F}_{e, e}^{(s)}$ and hence on $\mathcal{F}_{e}^{(s)}$. The connection between $\widehat{\mathfrak{s} \ell_{e}}$-weight spaces and blocks of the $q$-Schur algebras and hence blocks of $\operatorname{GL}(n, q)$ with $n \geq 0$ is known, and we describe it below. We denote the Weyl module of $\mathcal{S}_{q}(n)$ parametrized by $\lambda$ by $W(\lambda)$.

We need to introduce a function res on $\mathcal{P}$. If $\lambda \in \mathcal{P}$, the $e$-residue of the $(i, j)$-node of the Young diagram of $\lambda$ is the nonnegative integer $r$ given by $r \equiv j-i \bmod e$ for $0 \leq r<e$, denoted $\operatorname{res}_{i, j}(\lambda)$. Then $\operatorname{res}(\lambda)=\bigcup_{(i, j)}\left(\operatorname{res}_{i, j}(\lambda)\right)$.

Proposition. A weight space for $\widehat{\mathfrak{s} \ell_{e}}$ on $\mathcal{F}_{e}^{(s)}$ can be regarded as a union of blocks of $q$-Schur algebras with $q$ a primitive e-th root of unity.

Proof. The fact that res defines a weight space follows for instance from [Rouquier et al. 2013, p. 60]. Two Weyl modules $W(\lambda), W(\mu)$ are in the same block if and only if $\operatorname{res}(\lambda)=\operatorname{res}(\mu)$ (see for instance [Mathas 2004, Theorem 5.5, (i) $\Leftrightarrow$ (iv)].

Thus a weight space determines a set of partitions of a fixed $n \geq 0$.

Corollary. A weight space for $\widehat{\mathfrak{s} \ell_{e}}$ on $\mathcal{F}_{e}^{(s)}$ can be regarded as a union of blocks of groups $\operatorname{GL}(n, q)$, where the $n$ are determined from the weight space. 
We now have the following theorem which connects the $\ell$-decomposition numbers of $G_{n}$ with $n \geq 0$ with Fock spaces.

Theorem 8.1. Let $\phi_{\mu}$ be the Brauer character of $G_{n}$ indexed by $\mu \in \mathcal{P}_{n}$. Let $\lambda \in \mathcal{P}_{n}$. Then, for large $\ell,\left(\chi_{\mu}, \phi_{\lambda}\right)=\left(G^{-}(\lambda),|\mu\rangle\right)$.

Proof. The decomposition matrix of $\mathcal{S}_{q}(n)$ over a field of characteristic 0, with $q$ a root of unity, is known by Varagnolo-Vasserot [1999]. By their work the coefficients in the expansion of the $G^{+}(\lambda)$ in terms of the standard basis give the decomposition numbers for the algebras $\mathcal{S}_{q}(n)$ for $n \geq 0$, with $q$ specialized at an $e$-th root of unity.

By an asymptotic argument of Geck [2001] we can pass from the decomposition matrices of $q$-Schur algebras in characteristic 0 to those in characteristic $\ell$, where $\ell$ is large. Then by the Dipper-James theorem we can pass to the decomposition matrices of the groups $G_{n}$ over a field of characteristic $\ell$ with $q$ an $e$-th root of unity in the field.

Let $D_{n}$ be the unipotent part of the $\ell$-decomposition matrix of $G_{n}$ and $E_{n}$ its inverse transpose. Thus $D_{n}$ has columns $G^{+}(\lambda)$ and $E_{n}$ has rows given by $G^{-}(\lambda)$ (see [Leclerc and Thibon 1996, Section 4]). The rows of $E_{n}$ also give the Brauer characters of $G_{n}$, in terms of unipotent characters. These two descriptions of the rows of $E_{n}$ then give the result.

The following analog of Steinberg's tensor product theorem is proved for the canonical basis $G^{-}(\lambda)$ in [Leclerc and Thibon 1996].

Theorem 8.2. Let $\lambda$ be a partition such that $\lambda^{\prime}$ is e-singular, so that $\lambda=\mu+e \alpha$ where $\mu^{\prime}$ is e-regular. Then $G^{-}(\lambda)=S_{\alpha} G^{-}(\mu)$.

We now show that the rows indexed by partitions $\lambda$ as in the above theorem can be described by Lusztig induction. By replacing $S_{\alpha}$ by $\mathcal{L}_{\alpha}$ and using Theorem 6.1 it follows that in these cases, Lusztig-induced characters coincide with Brauer characters.

Theorem 8.3. Let $\lambda=\mu+e \alpha$ where $\mu^{\prime}$ is e-regular. Then, for sufficiently large $\ell$, the Brauer character represented by $G^{-}(\lambda)$ is equal to the Lusztig generalized character $R_{L}^{G_{n}}\left(G^{-}(\mu) \times \chi_{\alpha}\right)$, where $L=G_{m} \times \mathrm{GL}\left(k, q^{e}\right), n=m+k e$, and $\alpha \vdash k$.

By using the BMM bijection, Theorem 4.2, we have the following corollary.

Corollary. Let $\mu=\phi$, so that $\lambda=e \alpha$. Then the Brauer character represented by $G^{-}(\lambda)$ can be calculated from an induced character in a complex reflection group.

Some tables giving the basis vectors $G^{-}(\lambda)$ for $e=2$ are given in [Leclerc and Thibon 2000]. In our examples we use transpose partitions of the partitions in these tables, and rows instead of columns. 
We first give an example of a weight space for $\widehat{s l}_{e}$, which is also a block for $G_{n}$, with $n=4, e=4$. This is an example of a decomposition matrix $D$ for $n=4, e=4$. This matrix occurs in a paper of Ariki [2011] as a decomposition matrix of a $q$-Schur algebra.

$$
\left(\begin{array}{ccccc}
4 \| & 1 & 0 & 0 & 0 \\
31 \| & 1 & 1 & 0 & 0 \\
211 \| & 0 & 1 & 1 & 0 \\
1111 \| & 0 & 0 & 1 & 1
\end{array}\right)
$$

The following example is to illustrate Theorem 8.3. It was calculated using a GAP [2015] program for decomposition matrices of $q$-Schur algebras. It is an example of the inverse of a decomposition matrix for $n=6, e=2$. Here $\ell$ is large, because of the comparison with $q$-Schur algebras.

$$
\left(\begin{array}{rrrrrrrrrr}
1 & 0 & 0 & 0 & 0 & 0 & 0 & 0 & 0 & 0 \\
-1 & 1 & 0 & 0 & 0 & 0 & 0 & 0 & 0 & 0 \\
1 & -1 & 1 & 0 & 0 & 0 & 0 & 0 & 0 & 0 \\
-1 & 0 & -1 & 1 & 0 & 0 & 0 & 0 & 0 & 0 \\
-1 & 1 & -1 & 0 & 1 & 0 & 0 & 0 & 0 & 0 \\
1 & -1 & 1 & -1 & -1 & 1 & 0 & 0 & 0 & 0 \\
1 & 0 & 1 & -1 & -1 & 0 & 1 & 0 & 0 & 0 \\
0 & 0 & -1 & 1 & 1 & -1 & -1 & 1 & 0 & 0 \\
0 & 0 & 1 & -1 & 0 & 0 & 1 & -1 & 1 & 0 \\
0 & 0 & 0 & 0 & 0 & 0 & -1 & 1 & -1 & 1
\end{array}\right)
$$

Here the rows are indexed as: $6,51,42,41^{2}, 3^{2}, 31^{3}, 2^{3}, 2^{2} 1^{2}, 21^{4}, 1^{6}$. In the above matrix:

(1) The rows indexed by $1^{6}, 2^{2} 1^{2}, 3^{2}, 21^{4}, 41^{2}$ have interpretations as Brauer characters, in terms of $R_{L}^{G_{n}}$, with $L$ an $e$-split Levi of the form $\operatorname{GL}\left(3, q^{2}\right)$ for $\lambda=1^{6}, 2^{2} 1^{2}, 3^{2}$, of the form $\operatorname{GL}(2, q) \times \operatorname{GL}\left(2, q^{2}\right)$ for $\lambda=21^{4}$, and of the form $\operatorname{GL}(4, q) \times \mathrm{GL}\left(1, q^{2}\right)$ for $\lambda=41^{2}$.

(2) Put $L=\operatorname{GL}\left(3, q^{2}\right)$. Then:

(a) the row indexed by $3^{2}$ is $R_{L}^{G}\left(\chi_{3}\right)=\chi_{3^{2}}-\chi_{42}+\chi_{51}-\chi_{6}$,

(b) the row indexed by $2^{2} 1^{2}$ is $R_{L}^{G}\left(\chi_{21}\right)$ and

(c) the row indexed by $1^{6}$ is $R_{L}^{G}\left(\chi_{1^{3}}\right)$.

\section{Acknowledgement}

The author thanks Bernard Leclerc for valuable discussions on this paper, and the referee for comments and suggestions which have led to its improvement. 


\section{References}

[Ariki 2011] S. Ariki, “Graded $q$-Schur algebras”, preprint, 2011. arXiv 0903.3453v3

[Bezrukavnikov and Etingof 2009] R. Bezrukavnikov and P. Etingof, "Parabolic induction and restriction functors for rational Cherednik algebras", Selecta Math. (N.S.) 14:3-4 (2009), 397-425. MR 2010e:20007 Zbl 1226.20002

[Broué et al. 1993] M. Broué, G. Malle, and J. Michel, "Generic blocks of finite reductive groups", pp. 7-92 in Représentations unipotentes génériques et blocs des groupes réductifs finis, Astérisque 212, Société Mathématique de France, Paris, 1993. MR 95d:20072 Zbl 0843.20012

[Cabanes and Enguehard 2004] M. Cabanes and M. Enguehard, Representation theory of finite reductive groups, New Mathematical Monographs 1, Cambridge Univ. Press, 2004. MR 2005g:20067 Zbl 1069.20032

[Deligne 1990] P. Deligne, "Catégories tannakiennes", pp. 111-195 in The Grothendieck Festschrift, Vol. II, edited by P. Cartier et al., Progr. Math. 87, Birkhäuser, Boston, 1990. MR 92d:14002 Zbl 0727.14010

[Dipper and James 1989] R. Dipper and G. James, "The $q$-Schur algebra", Proc. London Math. Soc. (3) 59:1 (1989), 23-50. MR 90g:16026 Zbl 1197.90358

[Enguehard 1992] M. Enguehard, "Combinatoire des Symboles et Application de Lusztig", Publicationes du LMENS 92:1 (1992).

[Farahat 1954] H. Farahat, "On the representations of the symmetric group", Proc. London Math. Soc. (3) 4 (1954), 303-316. MR 16,11c Zbl 0058.25905

[Fong and Srinivasan 1982] P. Fong and B. Srinivasan, "The blocks of finite general linear and unitary groups", Invent. Math. 69:1 (1982), 109-153. MR 83k:20013 Zbl 0507.20007

[GAP 2015] The GAP Group, "GAP - Groups, Algorithms, and Programming, Version 4.7.8”, 2015, Available at http://www.gap-system.org.

[Garvan et al. 1990] F. Garvan, D. Kim, and D. Stanton, "Cranks and t-cores”, Invent. Math. 101:1 (1990), 1-17. MR 91h:11106 Zbl 0721.11039

[Geck 2001] M. Geck, "Modular Harish-Chandra series, Hecke algebras and (generalized) $q$-Schur algebras", pp. 1-66 in Modular representation theory of finite groups (Charlottesville, VA, 1998), edited by M. J. Collins et al., de Gruyter, Berlin, 2001. MR 2003f:20016 Zbl 0999.20008

[Lam 2005] T. Lam, "Ribbon tableaux and the Heisenberg algebra", Math. Z. 250:3 (2005), 685-710. MR 2006i:05175 Zbl 1066.05145

[Lascoux et al. 1997] A. Lascoux, B. Leclerc, and J.-Y. Thibon, "Ribbon tableaux, Hall-Littlewood functions, quantum affine algebras, and unipotent varieties", J. Math. Phys. 38:2 (1997), 1041-1068. MR 98c:05167 Zbl 0711.20007

[Leclerc and Thibon 1996] B. Leclerc and J.-Y. Thibon, "Canonical bases of $q$-deformed Fock spaces”, Internat. Math. Res. Notices 9 (1996), 447-456. MR 97h:17023 Zbl 0863.17013

[Leclerc and Thibon 2000] B. Leclerc and J.-Y. Thibon, "Littlewood-Richardson coefficients and Kazhdan-Lusztig polynomials", pp. 155-220 in Combinatorial methods in representation theory (Kyoto, 1998), edited by K. Koike et al., Adv. Stud. Pure Math. 28, Kinokuniya, Tokyo, 2000. MR 2002k:20014 Zbl 1058.20006

[Macdonald 1995] I. G. Macdonald, Symmetric functions and Hall polynomials, 2nd ed., Oxford University Press, New York, 1995. MR 96h:05207 Zbl 0824.05059

[Mathas 2004] A. Mathas, "The representation theory of the Ariki-Koike and cyclotomic $q$-Schur algebras", pp. 261-320 in Representation theory of algebraic groups and quantum groups, edited 
by T. Shoji et al., Adv. Stud. Pure Math. 40, Math. Soc. Japan, Tokyo, 2004. MR 2005f:20014 Zbl 1140.20003

[Rouquier et al. 2013] R. Rouquier, P. Shan, M. Varagnolo, and E. Vasserot, "Categorifications and cyclotomic rational double affine Hecke algebras", 2013. arXiv 1305.4456v1

[Shan and Vasserot 2012] P. Shan and E. Vasserot, "Heisenberg algebras and rational double affine Hecke algebras”, J. Amer. Math. Soc. 25:4 (2012), 959-1031. MR 2947944 Zbl 1287.20008

[Uglov 2000] D. Uglov, "Canonical bases of higher-level $q$-deformed Fock spaces and KazhdanLusztig polynomials", pp. 249-299 in Physical combinatorics (Kyoto, 1999), edited by M. Kashiwara and T. Miwa, Progr. Math. 191, Birkhäuser, Boston, 2000. MR 2001k:17030 Zbl 0963.17012

[Varagnolo and Vasserot 1999] M. Varagnolo and E. Vasserot, "On the decomposition matrices of the quantized Schur algebra”, Duke Math. J. 100:2 (1999), 267-297. MR 2001c:17029 Zbl 0962.17006

Received March 9, 2015. Revised October 12, 2015.

BHAMA SRINIVASAN

Department of Mathematics, Statistics, And Computer Science (MC 249)

UNIVERSITY OF ILLINOIS AT CHICAGO

851 SOUTH MORGan STREeT

CHICAGO, IL 60607-7045

UNITED STATES

srinivas@uic.edu 


\title{
PACIFIC JOURNAL OF MATHEMATICS
}

\author{
msp.org/pjm
}

Founded in 1951 by E. F. Beckenbach (1906-1982) and F. Wolf (1904-1989)

\section{EDITORS}

Don Blasius (Managing Editor)

Department of Mathematics

University of California

Los Angeles, CA 90095-1555

blasius@math.ucla.edu

\author{
Paul Balmer \\ Department of Mathematics \\ University of California \\ Los Angeles, CA 90095-1555 \\ balmer@math.ucla.edu \\ Robert Finn \\ Department of Mathematics \\ Stanford University \\ Stanford, CA 94305-2125 \\ finn@math.stanford.edu \\ Sorin Popa \\ Department of Mathematics \\ University of California \\ Los Angeles, CA 90095-1555 \\ popa@math.ucla.edu
}

\author{
Vyjayanthi Chari \\ Department of Mathematics \\ University of California \\ Riverside, CA 92521-0135 \\ chari@math.ucr.edu \\ Kefeng Liu \\ Department of Mathematics \\ University of California \\ Los Angeles, CA 90095-1555 \\ liu@math.ucla.edu \\ Jie Qing \\ Department of Mathematics \\ University of California \\ Santa Cruz, CA 95064 \\ qing@ cats.ucsc.edu
}

\section{PRODUCTION}

Silvio Levy, Scientific Editor, production@msp.org

\section{SUPPORTING INSTITUTIONS}

ACADEMIA SINICA, TAIPEI

CALIFORNIA INST. OF TECHNOLOGY

INST. DE MATEMÁTICA PURA E APLICADA

KEIO UNIVERSITY

MATH. SCIENCES RESEARCH INSTITUTE

NEW MEXICO STATE UNIV.

OREGON STATE UNIV.

\author{
STANFORD UNIVERSITY \\ UNIV. OF BRITISH COLUMBIA \\ UNIV. OF CALIFORNIA, BERKELEY \\ UNIV. OF CALIFORNIA, DAVIS \\ UNIV. OF CALIFORNIA, LOS ANGELES \\ UNIV. OF CALIFORNIA, RIVERSIDE \\ UNIV. OF CALIFORNIA, SAN DIEGO \\ UNIV. OF CALIF., SANTA BARBARA
}

\author{
Daryl Cooper \\ Department of Mathematics \\ University of California \\ Santa Barbara, CA 93106-3080 \\ cooper@math.ucsb.edu \\ Jiang-Hua Lu \\ Department of Mathematics \\ The University of Hong Kong \\ Pokfulam Rd., Hong Kong \\ jhlu@maths.hku.hk \\ Paul Yang \\ Department of Mathematics \\ Princeton University \\ Princeton NJ 08544-1000 \\ yang@math.princeton.edu
}

These supporting institutions contribute to the cost of publication of this Journal, but they are not owners or publishers and have no responsibility for its contents or policies.

See inside back cover or msp.org/pjm for submission instructions.

The subscription price for 2015 is US \$420/year for the electronic version, and \$570/year for print and electronic.

Subscriptions, requests for back issues and changes of subscribers address should be sent to Pacific Journal of Mathematics, P.O. Box 4163, Berkeley, CA 94704-0163, U.S.A. The Pacific Journal of Mathematics is indexed by Mathematical Reviews, Zentralblatt MATH, PASCAL CNRS Index, Referativnyi Zhurnal, Current Mathematical Publications and Web of Knowledge (Science Citation Index).

The Pacific Journal of Mathematics (ISSN 0030-8730) at the University of California, c/o Department of Mathematics, 798 Evans Hall \#3840, Berkeley, CA 94720-3840, is published twelve times a year. Periodical rate postage paid at Berkeley, CA 94704, and additional mailing offices. POSTMASTER: send address changes to Pacific Journal of Mathematics, P.O. Box 4163, Berkeley, CA 94704-0163.

PJM peer review and production are managed by EditFLOW ${ }^{\circledR}$ from Mathematical Sciences Publishers.

\section{PUBLISHED BY}

\section{mathematical sciences publishers \\ nonprofit scientific publishing}

http://msp.org/

(C) 2015 Mathematical Sciences Publishers 


\title{
PACIFIC JOURNAL OF MATHEMATICS
}

\author{
Volume 279 No. 1-2 December 2015
}

In memoriam: Robert Steinberg

Robert Steinberg (1922-2014): In memoriam V. S. VARADARAJAN

Cellularity of certain quantum endomorphism algebras

HENNING H. ANDERSEN, GUSTAV I. LEHRER and RUIBIN ZHANG

Lower bounds for essential dimensions in characteristic 2 via orthogonal representations ANTONIO BABIC and VLADIMIR CHERNOUSOV

Cocharacter-closure and spherical buildings

Michael Bate, Sebastian Herpel, Benjamin Martin and Gerhard RöHrLe

Embedding functor for classical groups and Brauer-Manin obstruction

Eva Bayer-Fluckiger, Ting-Yu LeE and Raman Parimala

On maximal tori of algebraic groups of type $G_{2}$

Constantin Beli, PhilipPe Gille and Ting-Yu LeE

On extensions of algebraic groups with finite quotient

MICHEL BRION

Essential dimension and error-correcting codes

SHANE CERNELE and ZiNOVy REICHSTEIN

Notes on the structure constants of Hecke algebras of induced representations of finite Chevalley groups

Charles W. CuRTis

Complements on disconnected reductive groups

FRANÇOIS DIGNE and JEAN MICHEL

Extending Hecke endomorphism algebras

Jie Du, Brian J. Parshall and LeOnard L. SCOTT

Products of partial normal subgroups

ELLEN HENKE

Lusztig induction and $\ell$-blocks of finite reductive groups

RADHA KESSAR and GUNTER MALLE

Free resolutions of some Schubert singularities

Manoj Kummini, Venkatramani Lakshmibai, Pramathanath Sastry and C. S. Seshadri

Free resolutions of some Schubert singularities in the Lagrangian Grassmannian

VenKatramani LAKSHMibai and ReUVEN HODGES

Distinguished unipotent elements and multiplicity-free subgroups of simple algebraic groups

Martin W. Liebeck, Gary M. Seitz and Donna M. Testerman

Action of longest element on a Hecke algebra cell module

GEORGE LUSZTIG

Generic stabilisers for actions of reductive groups

BENJAMIN MARTIN

On the equations defining affine algebraic groups

VLADIMIR L. POPOV

Smooth representations and Hecke modules in characteristic $p$

PETER SCHNEIDER

On CRDAHA and finite general linear and unitary groups

BHAMA SRINIVASAN

Weil representations of finite general linear groups and finite special linear groups PHAM HUU TIEP

The pro- $p$ Iwahori Hecke algebra of a reductive $p$-adic group, $\mathrm{V}$ (parabolic induction) MARIE-FRANCE VIGNÉRAS

Acknowledgement 